\title{
Warum gibt es Musik?
}

\author{
M. Spitzer, Ulm
}

„Dumme Frage!“ - werden viele Leser nach dem Überfliegen der Überschrift jetzt denken. „Sie machen doch selber gelegentlich solchen rhythmischen Lärm und werden daher wissen, dass das Spaß macht", werden manche dann vielleicht noch im Hinterkopf ergänzen. Dabei könnte man es belassen. Musik macht Freude und daher gibt es sie.

Betrachten wir eine ähnliche Frage: Warum gibt es Süßigkeiten? - Die Antwort „weil viele Menschen gerne Süßes essen“ befriedigt hier nur bei oberflächlicher Betrachtung, denn man kann sofort weiter fragen: „Und warum ist das so?", vielleicht mit dem Hintergedanken: „Wenn sie durch Süßigkeiten dick werden und dadurch früher sterben, müssten diejenigen Menschen mit einer besonderen Vorliebe für Süßes doch eigentlich längst ausgestorben sein." Sie sind es aber nicht! Wie kann das sein?

Jetzt sind wir nicht mehr bei Antworten wie "es macht Spaß 3 ", sondern fragen nach dem Grund dafür, dass etwas Spaß macht. Solche Warum-Fragen nach der "tieferen“ oder ultimativen Ursache - im Vergleich zur „oberflächlichen" oder naheliegenden Ursache (wie z. B.: „es macht Spaß“) kommen in der Biologie häufiger vor als beispielsweise in der Physik. Kaum jemand fragt: „Warum gibt es Gravitation?", und wenn, dann würden die meisten Physiker sagen: „Es gibt sie eben (schau den Apfel an, wie er vom Baum fällt oder den Mond, wie er sich um die Erde dreht) - basta“.

In der Biologie unterscheidet man schon lange zwei "Sorten“ von Ursachen: die naheliegende Ursache (Beispiel: „Menschen essen Süßes, weil es ihnen schmeckt") und die ultimative Ursache. ${ }^{1}$ Im Hinblick auf unser Beispiel könnte man die ultimative Ursache etwa wie folgt formulieren: „Menschen essen Süßes, weil die

Nervenheilkunde 2018; 37: 379-382

Korrespondenzadresse

Prof. Dr. Dr. Manfred Spitzer, Universitätsklinikum Ulm Klinik für Psychiatrie und Psychotherapie III

Leimgrubenweg 12, 89075 Ulm
Geschmacksqualität „süß“ das Vorhandensein von Zucker anzeigt und weil mit der Nahrung aufgenommener Zucker Energie zur Aufrechterhaltung lebensnotwendiger Prozesse im Körper bereit stellt. Die Rezeptoren für Süßes mitsamt der Vorliebe dafür sind Produkt der Evolution, d. h. sind durch zufällige Mutationen und natürliche Auswahl (Selektion) von Eigenschaften, Merkmalen, Prozessen oder Fähigkeiten entstanden, die dem Überleben dienten." Man spricht hier auch von "Anpassung", die nicht bewusst oder gar willensgesteuert funktioniert, sondern durch „blinde“ $\mathrm{Mu}$ tation und Selektion, dem Mechanismus der Evolution.

Zurück zur Musik und zur Frage, warum es sie gibt. Kein Geringerer als Charles Darwin ( $\$ Abb. 1), der Urvater der Evolutionstheorie, hat sich diese Frage gestellt. In seiner zweiten großen Schrift Zur Abstammung des Menschen (4) liest man Folgendes:

„Die Fähigkeit und Liebe zum Singen und zur Musik [...] darf hier nicht übergangen werden. Obschon die von Thieren aller Arten ausgestoßenen Laute vielen Zwecken dienen, kann doch mit Nachdruck hervorgehoben werden, dass die Stimmorgane ursprünglich in Beziehung zur Fortpflanzung der Art gebracht und vervollkommnet werden. Insecten und einige wenige Spinnen sind die niedrigsten Thiere, welche absichtlich einen Laut hervorbringen, und dies wird allgemein mit Hülfe sehr schön construierter Stridulationsorgane bewirkt, welche häufig allein auf die Männchen beschränkt sind. Die hierdurch hervorgebrachten Laute bestehen, wie ich glaube, in allen Fällen aus einem und dem nämlichen Tone, welcher rhythmisch wiederholt wird, und dies ist zuweilen selbst für das $\mathrm{Ohr}$ des Menschen angenehm. Ihr hauptsächlichs-

1 Meine Wortwahl ist bewusst der angloamerikanischen Literatur zu diesen Sachverhalten entnommen, wo man von „proximate cause“ und „ultimate cause" spricht. ter und in einigen Fällen ausschließlicher Nutzen scheint darin zu bestehen, entweder das andere Geschlecht zu rufen oder es zu bezaubern" (4, S. 640f).

Über Seiten gehen dann die Beschreibungen Darwins des Verhaltens von Kröten, Alligatoren, Singvögeln, Schildkröten, Gibbons, Walen, Mäusen im Hinblick auf lautliche Äußerungen vor allem der Männchen zur Paarungszeit. Er diskutiert in Anlehnung an Hermann von Helmholtz Konsonanz und Dissonanz (S. 643), argumentiert, und dass auch die Tiere „Vergnügen“ beim Singen bzw. Hören von Gesang haben (S. 644). „[...] denn wenn die Weibchen nicht fähig wären, solche Laute zu würdigen, und sie nicht von ihnen angeregt oder bezaubert würden, so würden die ausdauernden Anstrengungen der Männchen und die häufig nur ihnen allein zukommenden complicierten Gebilde nutzlos sein; und

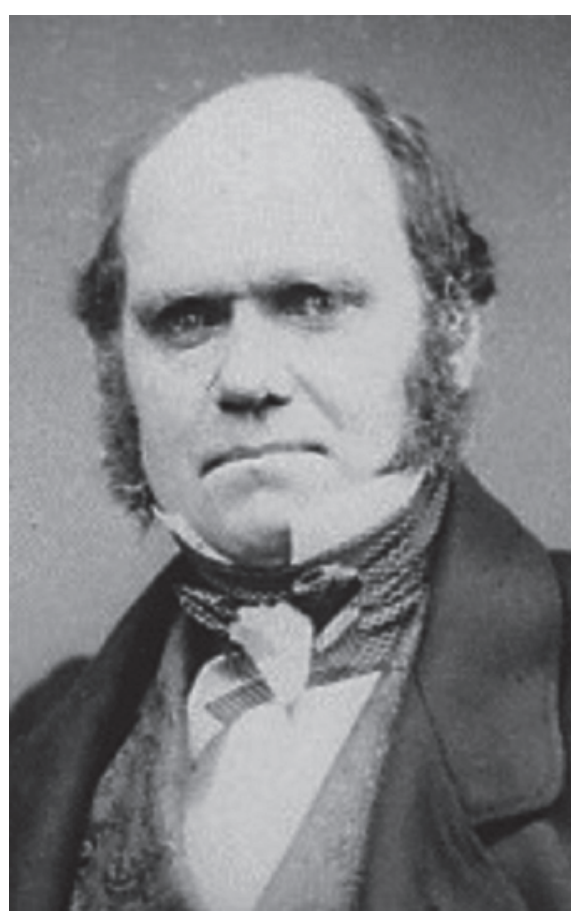

Abb. 1 Charles Darwin (1809-1882), Fotografie aus dem Jahr 1854, fünf Jahre vor der Publikation seines ersten großen Werks Die Entstehung der Arten (@gemeinfrei). 
dies kann man unmöglich glauben" (S. 644).

Für Darwin entstand die Musik im Verlauf der Menschheitsgeschichte vor der Sprache (S. 648; 18). In seinem Werk über die Entstehung der Arten kommt sie (noch) nicht vor, weil er sie - wie andere vollkommen „nutzlose“ Eigenschaften von Lebewesen (z. B. auffällige schmückende Körperteile wie Pfauenschwanz oder Geweih) letztlich Ergebnis von evolutionären Anpassungs- bzw. Optimierungsprozessen nicht erklären konnte: „Da weder die Freude an dem Hervorbringen musikalischer Töne noch die Fähigkeit hierzu von dem geringsten Nutzen für den Menschen in Beziehungen zu seinen gewöhnlichen Lebensverrichtungen sind, so müssen sie unter die mysteriösesten gerechnet werden, mit welchen er versehen ist," schreibt Darwin im Jahr 1871 (S. 644, Hervorhebung vom Autor, MS).

Wenn Musik so vollkommen nutzlos ist, wie konnte sie dann im Verlauf der Evolution entstehen?

Wenn Musik so vollkommen nutzlos ist, wie konnte sie dann im Verlauf der Evolution entstehen? Dieses für Darwin keineswegs randständige Problem der Entstehung von scheinbar völlig nutzlosen Strukturen (Pfauenschwanz) und Verhaltensweisen (lange Balz-Gesänge und/oder -Tänze bei Männchen vieler Vogelarten) veranlasste ihn letztlich zur Abfassung seines zweiten großen Werks, das mit vollständigem Titel „Die Abstammung des Menschen und die geschlechtliche Zuchtwahl" lautete. Hier diskutierte er neben der Evolution als Überleben des besser angepassten Organismus' (survival of the fittest) mit der sexuellen Selektion einen zweiten evolutionären $\mathrm{Me}$ chanismus, der kurz und knapp formuliert wie folgt funktioniert: Das Geschlecht mit dem höheren Reproduktionsaufwand - definitionsgemäß ist dies das weibliche Geschlecht ${ }^{2}$ - wählt zur sexuellen Reproduktion den Partner (und nicht umgekehrt), weil es mehr zu verlieren hat. Männchen konkurrieren also um die Gunst der Weibchen, und so kommt es zur Auswahl von Merkmalen beim Männchen, die auf besondere Qualitäten hinweisen.
Denkt man darüber zum ersten $\mathrm{Mal}$ nach, erscheinen männliche Größe und Stärke als Auswahlkriterien unmittelbar plausibel. Und in der Tat wird der bei vielen Arten zu beobachtende sexuelle Dimorphismus (Männchen und Weibchen sehen unterschiedlich aus) auf diese Weise erklärt. Aber damit längst nicht genug: auch „nutzlose“ Eigenschaften lassen sich erklären: Wenn sich ein männlicher Vogel (im grünen Urwald lebender) Vogel ein knallrotes Gefieder „leisten“ kann, muss er schon ein "toller Hecht" sein, denn die Farbe ist ja maximal auffällig und würde eigentlich seinen frühen Tod durch Fressfeinde verursachen. Das rote Kleid auf grünem Hintergrund ist also einerseits eine „Behinderung", die aber gerade dadurch anzeigt, wie fit der Junge ist, denn er kann sie sich ganz offensichtlich leisten (sonst wäre er nicht mehr da!). Feldstudien konnten zeigen, dass Weibchen prächtig farbig gefiederte Männchen bei der Paarung bevorzugen, weil die Männchen hierdurch eine höhere Qualität, beispielsweise bei der Aufzucht der Jungen, anzeigen: Zusätzliche Experimente ergaben, dass besonders bunt gefiederte Männchen dem Nest mehr Aufmerksamkeit schenkten und den Winter mit höherer Wahrscheinlichkeit überlebten (10).

Bunt gefiederte Männchen schenken dem Nest mehr Aufmerksamkeit und überleben den Winter mit höherer Wahrscheinlichkeit.

Dieses Handicap-Prinzip wurde in den 1970er-Jahren dem Wissenschaftlerehepaar Avishag (Pflanzenphysiologin) und Amotz (Zoologe) Zahavi publiziert und erklärt die Entwicklung von Merkmalen durch Partnerwahl, die einen Überlebensnachteil für den Träger bringen, aber als Signal die Qualität von dessen Genen belegen. Mittlerweile gibt es hierfür viele Bei-

2 Was die wenigsten wissen: ,weiblich“ ist biologisch nicht etwa als "Träger zweier X-Chromosomen“ definiert (die gibt es bei vielen Arten gar nicht), sondern als das Geschlecht mit dem höheren Reproduktionsaufwand. Dieser ergibt sich in vielen Fällen ganz einfach aus der Größe der Geschlechtszellen: Eizellen sind definitionsgemäß größer als Samenzellen. spiele, von denen zwei sehr hochrangig publizierte angeführt sein sollen (26):

1. Löwen (Panthera leo) mit schwarzer Mähne haben bei heftiger Sonne einen Nachteil (schwarz heizt sich stärker auf), sind aber bei Löwinnen begehrter (24).

2. Ägyptische Geier (Neophron percnopterus) haben ein sehr gelbes Gesicht, weil sie den Kot von Kühen fressen, der gelbe Karotenoidpigmente enthält (19). Das resultierende gelbe Gesicht signalisiert beispielsweise eine gute Immunabwehr und macht das Männchen daher für Weibchen besonders attraktiv.

Für Darwin war Musik das Paradebeispiel einer eigentlich "nutzlosen“ Funktion, die im Rahmen der sexuellen Selektion beim Menschen aus einfachen Anfängen der Lauterkennung zu immer größerer Perfektion des Hörens und Produzierens geführt hat.

Interessanterweise haben diese Überlegungen zu den Ursprüngen von Musik für mehr als hundert Jahre kaum Aufsehen erregt. Sie wurden kaum diskutiert. Dies änderte sich mit dem Aufkommen der Soziobiologie (25) und vor allem der evolutionären Psychologie (1) in den vergangenen Jahrzehnten: Musik als Ausdruck und Ergebnis der Selektion im Rahmen der Partnerwahl wurde $\mathrm{zu}$ einem Gemeinplatz (17). Aber stimmt das auch? Gibt es Musik wirklich nur, weil wir Menschen, ähnlich wie die Singvögel, balzen was das Zeug hält?

Musik als Ausdruck und Ergebnis der Selektion im Rahmen der Partnerwahl wurde zu einem Gemeinplatz.

Überall auf der Welt in jeder Kultur vorkommende Wiegenlieder weisen darauf hin, dass Musik auch Funktionen hat, die mit „Balz“ nichts zu tun haben: Wiegenlieder beruhigen, nehmen Ängste, signalisieren Kontakt und Präsenz (6, 21-23). Es geht bei ihnen zwar durchaus um social bonding, aber sicher nicht um Sex. Diese soziale Funktion von Musik trat in den vergangenen zwei Dekaden zunehmend in den Vordergrund. 
Schon vor knapp 20 Jahren schlug Björn Merker vom Institute for Biomusicology im schwedischen Ostersund vor, dass Musik eher durch synchrones Singen zur Verstärkung der Amplitude von Revierbegrenzungs-Lauten oder zur Signalisierung der Stärke einer Gruppe entstanden sei (16). Diese zunächst gewagte These reiht sich ein in seither zunehmend produzierte Arbeiten $\mathrm{zu}$ den gemeinschaftsstiftenden Auswirkungen von Musik.

Edward Hagen vom Institut für Theoretische Biologie der Humboldt Universität in Berlin publizierte im Jahr 2003 zusammen mit Gregory A. Bryant von der University of California in Santa Cruz die Überlegung, dass Musik und Tanz ein Organisations- und Kommunikationssystem darstellen, das der Kooperation von Mitgliedern verschiedener Gruppen diene. Sie hatten durch entsprechende Experimente herausgefunden, dass die die Synchronizität gemeinsam gemachter und wahrgenommener Musik die Wahrnehmung der Qualität der Musik und des Zusammenhalts der Gruppe beeinflusste. „Given the ubiquity of feasting and alliance-making in the ethnographic record, our hypothesis suggests that such "showoffs" may, in part, be demonstrating their ability to contribute to coalition quality [...] The payoff for the individual would be the improved quality of their coalition and, more importantly, obtaining an increased leadership role (and thus the ability to influence group actions); the payoff for group members would be increased coalition success in forming alliances and competing with other groups", schreiben die Autoren in ihrer Diskussion (28, S. 43).

Musik ist Teil vieler Feste, Rituale und anderer bedeutsamer gemeinschaftlicher Aktivitäten wie Gottesdienste, Hochzeiten oder Beerdigungen. Auch zur Jagd oder in den Krieg zeiht man mit „Musik und Tamtam“. Dies deutet stark daraufhin, dass Musik für den sozialen Zusammenhalt einer Gemeinschaft von entscheidender Bedeutung ist $(5,7,11,13)$. Hier reihen sich auch neueste Befunde zur Koordination und Kooperation ein (18) sowie zur Förderung von exekutiven Funktionen (12) und damit Emotionsregulation, was wiederum dem Miteinander sehr dienlich ist.

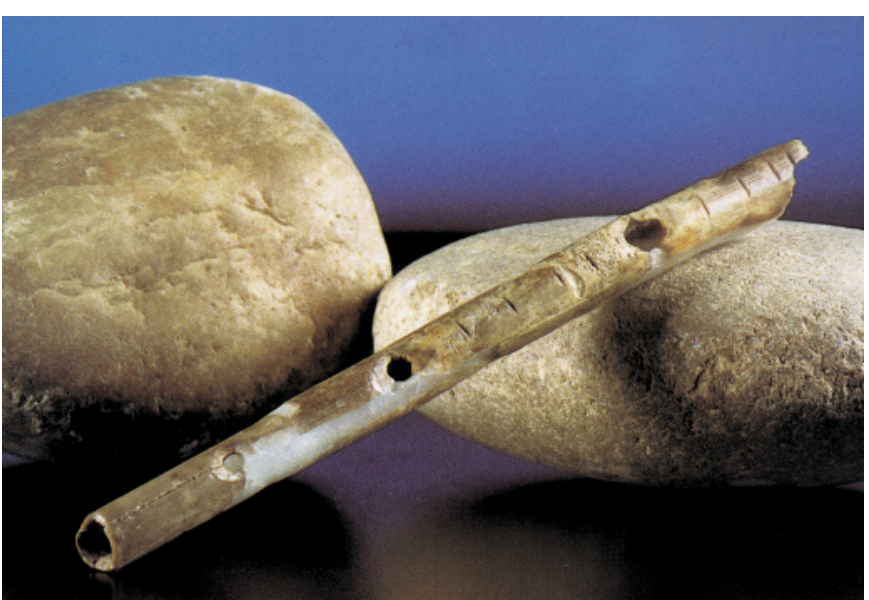

Dies deutet stark daraufhin, dass Musik für den sozialen Zusammenhalt einer Gemeinschaft von entscheidender Bedeutung ist.

Musikinstrumente gibt es seit mindestens 35000 Jahren (3) und wurden nicht nur unweit vom Wohnort des Autors ( $\triangleright$ Abb. 2) gefunden, sondern überall auf der Welt $(15,27)$. Das hat seinen Grund: Gemeinsames Singen, Musizieren und Tanzen fördert uns als Individuen und als Gemeinschaftswesen in ganz besonderer Weise, wie man es sonst vielleicht allenfalls noch vom Sport oder dem Theaterspiel her kennt. Zum Sport gibt es sehr viele empirische Befunde, zum Theater fast nichts und zur Musik glücklicherweise immer mehr. Dies ist wichtig, denn es gibt gerade in jüngster Zeit immer wieder Bestrebungen, ihren Stellenwert zu senken oder sie gar aus den Vorschulen oder dem Unterricht an Schulen ganz zu streichen. Dies hätte nach dem heutigen Stand des Wissens fatale Folgen für die gesunde Entwicklung der nächsten Generation.

\section{Literatur}

1. Barkow JH, Cosmides L, Tooby J (eds.) The adapted mind: Evolutionary psychology and the generation of culture. Oxford: University Press 1992.

2. Clayton M. The social and personal functions of music in crosscultural perspective. In: Hallam S, Cross I, Thaut M (eds.), Oxford handbook of music psychology, S. 35-44. Oxford: University Press 2009.
3. Conard NJ, Malina M, Münzel SC. New flutes document the earliest musical tradition in southwestern Germany. Nature 2009; 460: 737-740.

4. Darwin C. Zur Abstammung des Menschen (undatierter Nachdruck). Oldenbourg: Taschenbuch, Kirchheim 1971/1874.

5. Dissanayake E. Ritual and ritualization: Musical means of conveying and shaping emotion in humans and other animals. In: Brown S, Voglsten U (eds.) Music and manipulation: On the social uses and social control of music, S. 31-56. New York: Berghahn Books 2006.

6. Fernald A. Intonation and communicative intent in mothers' speech to infants: Is the melody the message? Child Development 1989; 60: 1497-1510.

7. Fitch WT. The biology and evolution of music: a comparative perspective. Cognition 2006; 100: 173-215.

8. Hahn J. Neue besondere Funde aus dem Geißenklösterle und dem Hohle Fels. Eine 36.000 Jahre alte Knochenflöte aus dem Geißenklösterle. In: A. Scheer (Hrsg.), Höhlenarchäologie im Urdonautal bei Blaubeuren, S. 87-89. Urgeschichtliches $\mathrm{Mu}$ seum, Blaubeuren 1994.

9. Haidt J, Seder JP, Kesebir S. Hive psychology, happiness, and public policy. Journal of Legal Studies 2008; 37: 133-S156.

10. Hill GE. Plumage coloration is a sexually selected indicator of male quality. Nature 1991; 350: 337-339.

11. Huron D. Is music an evolutionary adaptation? Annals of the New York Academy of Sciences 2001; 930: 43-61.

12. Kammer T, Spitzer M. Macht Musizieren wirklich schlau? Transfereffekte auf kognitive Leistungen. Nervenheilkunde 2018; 37: 392-397.

13. Kirschner S, Ilari B. Joint drumming in Brazilian and German preschool children: cultural differences in rhythmic entrainment, but no pro-social effects. J Cross Cult Psychol 2014; 45: 137-166.

14. Kirschner S, Tomasello M. Joint drumming: social context facilitates synchronization in preschool children. J Exp Child Psychol 2009; 102: 299-314.

15. Kuney D, Turk I. New perspectives on the beginnings of music: Archeological and musicological analysis of a middle paleolithic bone „flute“. In: 
Wallin NL, Merker B, Brown S (eds.) The origins of music. Cambridge, MA: MIT Press 2000; 235-268.

16. Merker B. Synchronous chorusing and the Origins of music. Musicae Scientiae (Special Issue 1999-2000): 59-73.

17. Miller GF. Evolution of human music through sexual selection. In: Wallin NL, Merker B, Brown S (eds.) The origins of music. Cambridge: MIT Press 2000; 329-360.

18. Mithen S. The singing neanderthals: The origins of music,language, mind, and body, London: Weidenfeld \& Nicholson 2005.
19. Negro JJ, Grande JM, Tella JL, Garrido J, Hornero D, Donázar JA, Sanchez-Zapata JA, BenItez JR, Barcell M. Coprophagy: an unusual source of essential carotenoids. Nature 2002; 416: 807-808.

20. Puts DA et al. Sexual selection on male vocal fundamental frequency in humans and other anthropoids. Proc R Soc B 2016; 283: 20152830.

21. Spitzer M. Musik im Kopf. Stuttgart: Schattauer 2002/2014

22. Spitzer M. Musik: Von der Koordination zur Kooperation Prosoziale Effekte von Musizieren, Singen und Tanzen. Nervenheilkunde 2018; 37: 433-438.
23. Trehub SE. Musical predispositions in infancy. Annals of the New York Academy of Sciences 2001; 930: 1-16.

24. West PM, Packer C. Sexual selection, temperature, and the lion's mane. Science 2002; 297: 1339-1343.

25. Wilson EO. Sociobiology: The new synthesis. Harvard: University Press 1975

26. Zahavi A, Zahavi A. The Handicap Principle. Oxford: University Press 1997.

27. Zhang J, Harbottle G, Wang C, Kong Z. Oldest playable musical instruments found at Jiahu early neolithic site in China, Nature 1999; 401: 366-368.

28. Hagen EH, Bryant GA. Music and dance as a coalition signaling system. Hum Nat 2003; 14: 21-51. 